\title{
Enriching complex road intersections from OSM with traffic- related behavioral information
}

\author{
Andreas Keler ${ }^{\mathrm{a},}$ *, Georgios Grigoropoulos ${ }^{\mathrm{a}}$, Dominik Mussack ${ }^{\mathrm{a}}$ \\ ${ }^{a}$ Chair of Traffic Engineering and Control, Technical University of Munich, Munich, Germany; andreas.keler@tum.de, \\ george.grigoropoulos@tum.de,dominik.mussack@tum.de \\ * Corresponding author
}

\begin{abstract}
Bicycle traffic in urban environments has various complex aspects in relation to the used space and the assigned transport infrastructures, especially in urban environments. Depending on the traffic situations, the built static urban infrastructure design, and, the other traffic participants involved, there are more or less challenging possibilities of its representation. Modelling and simulating urban bicycle traffic should include the interactions to other traffic participants, and, of course, behavioral aspects within each ride. Therefore, we introduce a method for enriching road features from OSM with traffic-related behavioural information. We focus on road intersections, since those are the locations with the highest number of interactions, communications, and, accidents. Our aim is to extend microscopic traffic flow simulations for achieving higher accuracies in simulation results and for applying bicycle movement models that are more adapted to reality. As an example we present a behavioral catalogue for cyclists, focussing on urban roundabouts, which results from real video observations and subsequent classifications. We classify into typical, frequently occurring and unusual bicycle maneuvers, and, include those into a specification procedure when enriching various OSM features.
\end{abstract}

Keywords: Traffic maps, Microscopic traffic flow, Traffic simulation, Interaction and communication, Traffic situations, Travel behavior, Turning maneuvers, Vulnerable road users

\section{Introduction}

Microscopic traffic flow simulations often rely on virtual road network representations with sub-lane accuracies. Nevertheless, it is challenging to simulate bicycle traffic, since bicyclists do not only make use of the bicycle traffic infrastructure, but also participate in mixed traffic, as well as on pedestrian areas. Depending on specific infrastructural designs, especially at road intersections (crossings and roundabouts), bicyclists prefer one option over another. Following on this assumption, we can define typical, frequently occurring and unusual maneuvers of bicyclists for specific infrastructural design elements.

The idea of the present approach is not only to model urban road infrastructure in an accurate way for guaranteeing realistic traffic simulation results, it is also the inclusion of additional fictional arcs or road elements within the modeling process.

One advantage of this procedure, is achieving a simple approach of providing more realism into microscopic traffic flow simulation. Therefore, the mapped environment (e.g. one complex urban crossing) should be subdivided into (1) visible and physically present elements of the spatial cutout of urban environment that has been modelled with high spatial accuracy, and, (2) line shapes that represent specific maneuvers of traffic participants (in our case bicyclists). Via the second options we might imply traffic-related behavioral information into the simulation, coming from traffic data acquisitions, video recordings or simply local knowledge.
The outcomes of the catalogue will be exemplarily presented for an urban roundabout situated in the outskirts of Munich, Germany.

\section{Representation possibilities for transport infrastructural features}

Another challenge after the extraction of accurate map information, as from OSM, is the representation or behavioral information a specific spatial features. This is connected with suitable data formats that allow more or less the inclusion of semantical information, which might be complex, especially when focusing of traffic-related information.
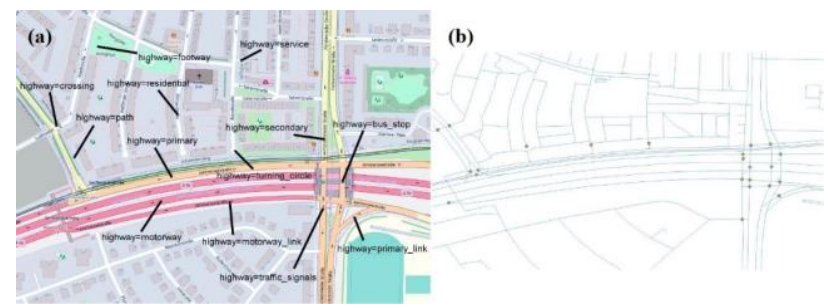

Figure 1. Cutout of from OSM of a part of the inner City of Munich with (a) different values for the attribute highway of specific features, and, (b) extracted road network information that is being used for modeling and enriching of urban infrastructure with behavioral information.

Parts of these semantical information, especially in urban environments, are often already mapped within an acceptable spatial accuracy, and, are growing since occurrence of the OSM initiative in 2004. Figure 1a shows 
a cutout of the inner City of Munich with semantics connecting road types to specific features. This is the base for our modeling and enrichment process. Figure 1b shows the extracted information from the same area that we are using for adding behavioral information. Besides this, it is even possible to connect data on historical accidents with specific features.

\section{Testing methods for the realistic representation of bicyclist behaviour - the case of data enrichment at urban roundabouts}

The general aim of the presented approach is achieving a detailed representation of the complex behavior of vulnerable road users, which might benefit autonomous or automated vehicle in interacting with its static (infrastructural design) and dynamic (traffic participants) environment. During our analyses from bird view trajectories at intersections via video devices, we have already found different aspects that have to be included into this specification: complex interaction and communication patterns between vehicle drivers, bicyclists and pedestrians are directly dependent on the infrastructural designs, traffic demands, changing visibilities and external environmental influences such as varying weather conditions.

Focusing on the infrastructural design, we have classified 4 general types of road infrastructure components: connection roads, crossings, $\mathrm{T}$-junctions and roundabouts. The procedure of enriching the latter is pictured in Figure $2 \mathrm{a}$ to $2 \mathrm{~d}$.

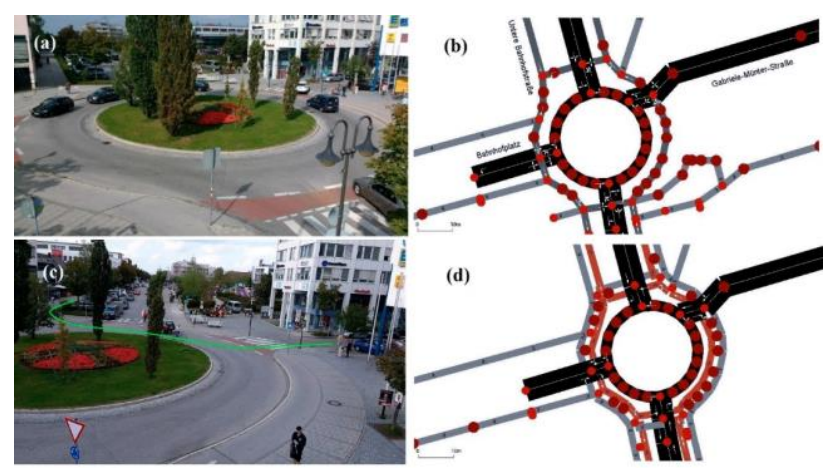

Figure 2. Modeling and enriching an urban roundabout with (a) photograph of the present state of the roundabout, (b) its modelled extraction from OSM, (c) representation of a typical bicyclist behavior via polyline, and, (d) inclusion of the specific behavior as possible (non-visible) maneuver via SUMO (Lopez et al. 2018).

Important is also the support of representing the road network up to the lane level, as it is supported by the OpenDRIVE standard. This allows us to visualize the road infrastructure in a realistic three-dimensional manner, as it is done for bicycle simulator studies (Keler et al. 2018).

\section{Designing a catalogue for the derivation of possible behavioral information}

The design of our catalogue on possible behavioral information of cyclist relies on real observations in urban environments. Depending on the time of the day and the selected observation time window, we can extract more or less real data that might be empirically analysed. As pictured in Figure 2, we focus on a small roundabout in Untere Bahnhofstraße, Germering (Germany), where cyclists have an important role in the usual interactions in daily traffic. Our catalogue partition on roundabouts consists of 13 defined maneuvers in Table 1 and in Table 2. While Table 1 shows the typical and highly frequent maneuvers, Table 2 shows the unusual bicycle maneuvers, which are still observable on several days.

Table 1 implies the expected maneuvers in the catalogue numbers from 10.1 to 10.4 that are compliant to traffic rules.

\begin{tabular}{|l|l|}
\hline Doscription \\
\hline 10.1
\end{tabular}

Table 1. Possible behavior of cyclists at a small roundabout in Untere Bahnhofstraße, Germering (Germany) with typical, and, highly recurrent maneuvers.

Maneuvers 11.1 and 11.2 in Table 1 show highly recurrent maneuvers that often imply violations of pedestrian transport infrastructure such as pedestrian crossings or sidewalks. When it comes to unusual behaviour as represented in Table 2, we often have more complex situations that are caused by numerous different traffic participants. One important attribute or numerous attributes is the traffic flow rate, which can be related to the capacity of the investigated roundabout. 


\begin{tabular}{|l|l|} 
Description \\
- Unusual behavior \\
- Cyclist is turning left by \\
driving along circular \\
oadway in the opposite \\
direction
\end{tabular}

Table 2. Possible behavior of cyclists at a small roundabout in Untere Bahnhofstraße, Germering (Germany) with unusual maneuvers.

Every entry and exit of the roundabout, and, consequently the circular roadway are influenced by a specific traffic flow rate value. Therefore, most of the 7 maneuvers in
Table 2 are dependent on the varying presence and volumes of other traffic participants. Similar to Table 1, Table 2 has strategical aspects of how cyclists plan their route. There are often aims to achieve the shortest path, which comes along with violating assigned space of pedestrians and vehicle drivers.

\section{Specification of bicyclist maneuvers}

For the case of urban roundabouts, there are in total 4 different bicycle maneuver classes:

- Passing the roundabout;

- Usage of sidewalk;

- Usage of pedestrian crossing;

- Passing in wrong direction;

- Usage of side space.

Depending on the number of types per each class, which is ranging between 2 and 4, it is possible to define static and dynamic attributes of respective components.

\section{Acknowledgements}

The presented research was conducted within the ongoing research project @CITY (Automated Vehicles and Intelligent Traffic in the City), partial project 3 on concepts and pilot applications, funded by the BMWI (Federal Ministry for Economic Affairs and Energy).

\section{References}

Keler, A., Kaths, J., Chucholowski, F., Chucholowski, M., Grigoropoulos, G., Spangler, M., Kaths, H. and Busch, F. (2018). A bicycle simulator for experiencing microscopic traffic flow simulation in urban environments. 2018 21st International Conference on Intelligent Transportation Systems (ITSC), IEEE, 3020-3023.

López, P. A., Behrisch, M., Bieker-Walz, L., Erdmann, J., Flötteröd, Y.-P., Hilbrich, R., Lücken, L., Rummel, J., Wagner, P. and Wießner, E. (2018). Microscopic Traffic Simulation using SUMO. 2018 21st International Conference on Intelligent Transportation Systems (ITSC), IEEE, 2575-2582. 